gewinnt damit wieder den Anschluß an die übrigen Ascomyceten.

Perisporium funiculatum Preuß gehört zur Familie der Perisporiaceen, Magnusia nitida Sacc. wird heute zu den Plectascineen gerechnet; früher
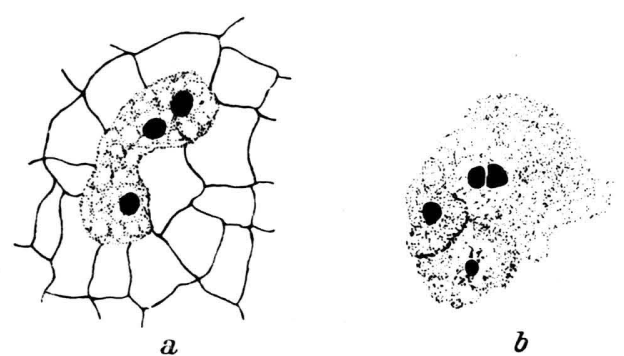

Abb. 8. Schnitte durch junge Perithezien. a: Dicke, unseptierte Hyphe mit einem Kernpaar und einem Einzelkern; b: eine 2-kernige Zelle und zwei je 1-kernige Zellen (vgl. Text). Vergr. 1040/1.

war diese Form ebenfalls den Perisporiaceen zugeordnet.

Allen drei Formen, Magnusia nitida, Perisporium funiculatum und Sphaerotheca mors uvae, ist gemeinsam, daß das Oogonium mehrere zweikernige Zellen liefert. (Bei den Laboulbeniaceen kommen ebenfalls mehrere zweikernige Zellen vor. Diese Gruppe sei hier aber außer acht gelassen!) Während die Zellteilungen zum Stillstand kommen, gehen die Kernteilungen weiter. Es entstehen so mehrere vielkernige Ascogone, aus denen die ascogenen Hyphen entsprossen, die wiederum die Asci liefern.

Bei Sph.m.u. wird aber bekanntlich nur ein Ascus gebildet, und nachdem sich das Oogonium in mehrere zweikernige Zellen geteilt hat, scheint es dem Pilz auf einmal ,bewußt“ zu werden, daß er eine ganz falsche Entwicklung eingeschlagen hat. Die Zellgrenzen werden wieder aufgelöst und die Kerne degenerieren bis auf ein Paar. Durch Verschmelzen dieser beiden Kerne entsteht der primäre Ascuskern. Die Bildung ascogener Hyphen ist unter diesen Umständen überflüssig.

Das abweichende Verhalten von Sph.m.u. ist zweifellos darauf zurückzuführen, daß nur noch ein Ascus im Fruchtkörper zur Ausbildung gelangt. Die vergleichende Gegenüberstellung der beiden Gattungen Perisporium und Sphaerotheca läßst wohl die Schlußfolgerung zu, daß es sich bei Perisporium um einen ursprünglichen und bei Sphaerotheca um einen abgeleiteten Typ handelt. Noch weitere Folgerungen aus den gewonnenen Ergebnissen zu ziehen, erscheint verfrüht.

\title{
Zwischenhirnstudien II
}

\author{
Von Rudolf BachmanN \\ Aus dem Anatomischen Institut der Universität Göttingen \\ (Z. Naturforschg. 3 b, 51-55 [1948]; eingegangen am 3. Februar 1948)
}

\begin{abstract}
An Hand der Übertragung von C a s p e r s s o n s Ultraviolett-Absorptions-Messungen auf das lichtmikroskopische Bild von Nervenzellkernen wird am Zwischenhirn der Maus ein quantitatives Verfahren (Differentialnervenzellbild) entwickelt. Dies gestattet eine schärfere Charakterisierung der einzelnen $Z_{w}$ ischenhirnareale nach dem Zellkernbild (Cytoarchitektonik). Verglichen werden Ergebnisse an Areal 6 und 9 nach Gr ünth a l. Die Möglichkeit der Verwendung dieser morphologischen Methode zur funktionellen Deutung von Zwischenhirngebieten wird diskutiert.
\end{abstract}

Coll in der Hirnforschung ein bestimmtes Areal des Zentralorgans morphologisch abgegrenzt werden, so stehen verschiedene bewährte Untersuchungsmethoden zur Verfügung. In erster Linie werden wir versuchen, über die Cytoarchitektonik den in Frage kommenden Komplex zu klären, d.h. wir gehen aus von der Art der Anordnung der Nervenzellen, der Dichte ihrer Lage- rung, ihrer Größe und Form, dem Verhalten ihrer Fortsätze, ihrer Nissl-Substanz im Cytoplasma. Von den Methoden der Myeloarchitektonik, Glioarchitektonik, Angioarchitektonik usw. sei hier abgesehen.

Auch im Bereich des Zwischenhirnes steht an der Spitze der morphologischen Untersuchung die cytoarchitektonische Aufgliederung. Sie ist bei 
Mensch und Tier bereits weit vorangetrieben, und es gelingt zumeist, 20-30 und mehr einzelne Areale allein aus dem Hypothalamus des Diencephalon herauszuarbeiten.

Unsere eigenen Untersuchungen gehören in den Rahmen der Cytoarchitektonik; diese soll in zwei Richtungen vertieft und erweitert werden:

1. durch eine schärfere Charakteristik der Zellkernverhältnisse, ganz bésonders des Nucleolarchromatins in einem gegebenen Areal. Insoweit sprechen wir von Karyoarchitektonik als einer Unterabteilung der Cytoarchitektonik.

2. durch eine quantitative Auswertung der Kernbefunde, indem pro Areal 200 Nervenzellen nach einem tabellarischen Schema bearbeitet werden. Insofern sprechen wir von einem Differentialnervenzellbild, etwa in Analogie zum Differentialblutbild der Hämatologen.

Die Methode sei in folgender Weise entwickelt:

1. Die Anschauung, daß das Kernkörperchen lediglich den Stapelplatz wertloser Exkrete des Kernstoffwechsels darstelle, muß revidiert werden ${ }^{1}$. Besonders im Eiweiß-Stoffwechsel des Kernes und der Zelle scheint der Nucleolus eine wesentliche Rolle $\mathrm{zu}$ spielen.

2. Dies zeigt sich nun morphologisch darin, daß wir keineswegs immer das Kernkörperchen in der stereotypen Form einer kleinen Kugel nach Art unserer Lehrbuchabbildungen vorfinden; das Gebilde kann recht absonderliche Formen aufweisen (Abb. 1)*.

3. Verständnis für derartige Nucleolarbilder haben uns die Arbeiten von Casperss on und seinen Schülern gebracht.

a) Die von Caspersson (s. z. B. Hydén²) entwickelte Apparatur erlaubt absorptionsspektrale Messungen im ultravioletten Licht noch an Objekten in der Größenordnung von $1 \mu$. Aus dem Spektrum kann der Gehalt an UV-absorbierenden Substanzen berechnet werden. Im mittleren UVWellenlängenbereich beherrschen Nucleinsäuren und Eiweißkörper die Absorption. Aus der Absorptionskurve eines Zellbestandteiles (z. B. des Nucleolus, des Raumes zwischen Nucleolus und Kernwand, der Kernwand usw.) ist ihr Gehalt an Nucleinsäuren, Tyrosin, Tryptophan, Hexonbasen usw. berechenbar.

b) Caspers ons Ergebnisse: Der Nucleolus besteht aus diaminosäurereichen Proteinen so-

* Abb. 1-3 s. Tafel S. 48 a.

1 C. u. O. Vo g t, Ärztl. Forschg. 1, 8 u. 43 [1947]. wie aus geringen Mengen von Ribosenucleotiden. Er zeigt temporäre Vergrößerung und Vermehrung seiner Nucleotide. In solchen Momenten erfolgt ein Abstrom der diaminosäurereichen Proteine zur Kernmembran, Kondensation dieser in und außen auf der Kernmembran als feulgennegative Kernmembrannucleotide.

c) Die vom Nucleolus gegen die Kernmembran abströmenden Produkte bilden die sogenannte Anlagerung (Abb.2, früher oft als Kernfalten mißdeutet, zuerst richtiggestellt von H.Spatz), der von der Kernmembran temporär festgehaltene Teil die sogenannte Einlagerung und die außen auf der Kernmembran liegenden Bestandteile eine Auflagerung. Wir selbst fügen diesen Begriffen noch den einer Auslagerung ins Cytoplasma hinzu.

d) Ca spers s on s Schüler Hy dén - L a n d ström wies nun nach, daß diese mit der angegebenen indirekten Untersuchungsmethode erhobenen Befunde auf die Verhältnisse des NisslPräparates und auf die Beobachtung im gewöhnlichen Lichtmikroskop vollkommen übertragen werden können. Dies wurde inzwischen von C. und $\mathrm{O}$. $\mathrm{Vog} \mathrm{t}^{1}$ bestätigt. Unseren eigenen Untersuchungen liegen Schnittserien durch das Gehirn der weißen Maus zugrunde, die mit Molybdänhämatoxylin, einem bei richtiger Differenzierung exquisiten Kernfarbstoff, gefärbt worden sind. An diesen wollen wir nunmehr, unter Berücksichtigung der Ergebnisse von C a s pers s o n, unser Differentialnervenzellbild aufstellen. Es heißt dies also, daß mittels eines Utbergangs aus der qualitativen in die quantitative Untersuchungstechnik der Zustand des Nucleolarchromatins eines jeden Zellkernes systematisch (neben den übrigen Befunden an Kern und Plasma) tabellarisch erfaßt werden soll.

Die Aufstellung des Differentialnervenzellbildes erfolgt in den folgenden Schritten:

1. Einstellung des zu untersuchenden Areales. Zur Benennung der Areale benutzen wir (trotz bekannter Bedenken!) die Gliederung des Hypothalamus der Maus nach Gr ü $\mathrm{th}$ a ${ }^{3}$.

Einstellung einer Ausgangsstelle. Mittels Kreuztischverschiebung wird von dieser aus nach links oder rechts, nach oben oder unten gegangen; alle etwa in die Mitte des Gesichtsfeldes geratenden Zellen bzw. Zellkerne werden untersucht; nur insofern sie nicht vollständig angeschnitten erscheinen, werden sie weggelassen und durch die nächste Zelle ersetzt.

2 H. Hy dén, Z. mikrosk. anat. Forschg. 1943, 54. ${ }^{3}$ E. Grünthal, Arch. Psychiatrie 90, 216 [1930]. 
2. Festlegung des Zelltypes. Erfahrungsgemäß lassen sich in jedem Areal nach Kern und Plasma meist mehrere Zelltypen unterscheiden. Auf diese interessante Frage kann hier nicht näher eingegangen werden; zur Verdeutlichung dessen, was bei dieser Typisierung etwa zur Entscheidung steht, sei auf Abb. 3 verwiesen, wo aus Areal 6 der Maus (Nucl. infundibulomamillaris) unmittelbar nebeneinander links Zelltyp I, rechts Zelltyp II zu beobachten ist.

3. Kernmessung: Messung des kleinsten und größten Durchmessers der Zelle und Berechnung des arithmetischen Mittels aus beiden (mittlerer Durchmesser).

4. und 5. Beurteilung des Cytoplasmas: Notieren wir zu der untersuchten Zelle Ziffer 4, so bedeutet dies ein dunkles, an Nissl-Substanz relativ reiches Cytoplasma; Ziffer 5 bedeutet helles, an Nissl-Substanz armes Plasma [vgl. Zelltyp I (4) und II (5) der Abb. 3]. 4/5 gilt für Mischformen und Grenzfälle.

6. bis 9. Allgemeine Beurteilung des Nucleolarchromatins:

6. Das Nucleolarchromatin ist in Form eines (oder mehrerer) groben, scharf begrenzten, konzentrierten Nucleolus allein im Zellkern enthalten.

(Zur Frage der Abgrenzung von Nucleolarchromatin und Chromosomenchromatin: In reifen Nervenzellen, die ihre Teilungsfähigkeit praktisch eingebüßt haben, in denen also der chromosomale Apparat sicher von anderer (um nicht zu sagen geringerer) Bedeutung ist als in teilungsfähigen Zellen, hebt sich bei der von uns verwendeten Färbung der Nucleolarapparat scharf vom Chromatinrest ab. Daß indessen bereits alle Verwechslungsmöglichkeiten ausgeschlossen seien, wollen wir nicht behaupten.)

7. Das Nucleolarchromatin erscheint in kleine Bruchstücke zersplittert (emittiert), die gewöhnlich in Nähe der Kernmembran liegen.

8. Der Nucleolarapparat befindet sich in Emission, d.h. an Stelle des konzentrierten Kernkörperchens findet sich ein mehr oder weniger aufgelockertes Gebilde, das helle Flecken (Vakuolen) enthält, das keine scharfe Begrenzung mehr aufweist, oder das Fortsätze, die sich in der Färbungsintensität vom zentralen Stück des Nucleolus unterscheiden, in den Kernraum oder gegen die Kernwand richtet (s.o. „Anlagerung“).

9. Unbestimmbarkeitsfall.

10. bis 30. Einzelbeurteilung des Nucleolarchromatins, speziell der Lagerung seiner Teile in bezug auf die Kernmembran. Jeder Lagerungstypus wird wiederum durch eines der Symbole (10-30) charakterisiert.

In dieser Weise werden pro Areal 200 Zellen untersucht. Das Ergebnis jst eine Übersicht, wie sie in Tab. 1 in einem Teilausschnitt wiedergegeben ist. Es kann nicht die Aufgabe dieser kurz informierenden Notiz sein, die cytologische Bedeutung aller gebrauchten Symbole durchzusprechen. Ein Beispiel mag genügen. Die zu Zelle 98 in der

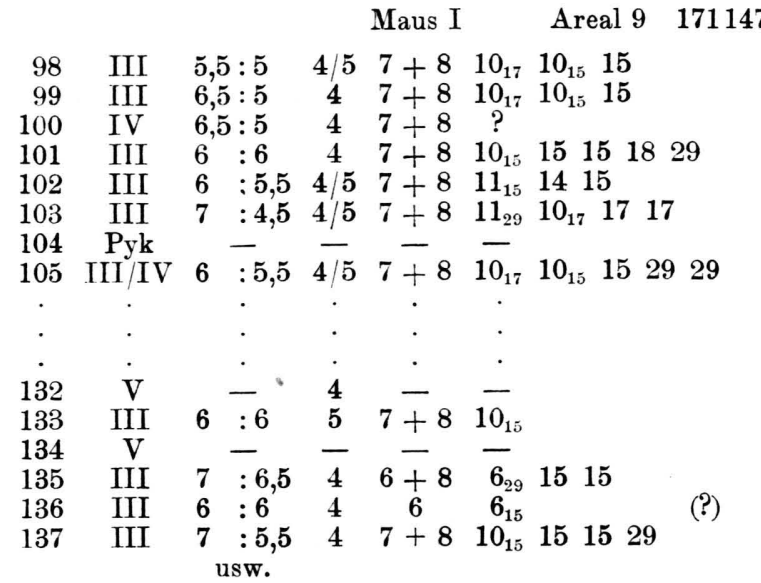

Tab. 1. Differentialnervenzellbild. Erklärung im Text.

Tab. 1 niedergeschriebenen Symbole bedeuten: Die Zelle gehört zum Zelltyp III und ihr Kern mißt 5,5:5 Teilstriche (ein Teilstrich entspricht

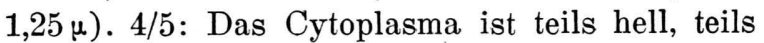
dunkel. $7+8$ : Das Nucleolarchromatin ist teilweise zersplittert (emittiert), liegt aber andererseits in einer Emission vor. $10_{17}$ : Diese Emission hat noch nicht zu einer vollständigen Zergliederung des Nucleolus geführt, jedoch geht eine in der Farbintensität von ihm unterscheidbare Substanz vom Nucleolus aus; der Index 17 besagt, daß diese Substanz in Form einer Anlagerung + Einlagerung + Auflagerung Beziehung zur Kernmembran aufgenommen hat. 10, Eine zweite Emission hat (Index 15) lediglich die Kernmembran erreicht (Anlagerung + Einlagerung), ohne diese zu durchsetzen. 15: Ein emittiertes Bröckel-

\begin{tabular}{|c|c|c|c|c|c|c|}
\hline \multirow{2}{*}{$\sum^{\infty}$} & \multirow{2}{*}{$\begin{array}{l}\vec{\Phi} \\
\vdots \\
\end{array}$} & \multicolumn{5}{|c|}{$\mathrm{Z}$ e $11 \mathrm{t}$ y } \\
\hline & & I & II & III & IV & V \\
\hline \multicolumn{7}{|c|}{ a) Vergleich der Hauptzelltypen } \\
\hline I & 6 & 94 & 60 & 17 & 15 & 9 \\
\hline II & 6 & 105 & 46 & 11 & 16 & 8 \\
\hline III & 6 & 94 & 43 & 8 & 27 & 7 \\
\hline I & 9 & - & - & 111 & 34 & 25 \\
\hline \multicolumn{7}{|c|}{ b) Vergleich der Cytoplasmaverhältnisse } \\
\hline I & 6 & 711112 & 3156 & 485 & $\begin{array}{llll}3 & 0 & 11_{(1)}\end{array}$ & $\begin{array}{lll}0 & 15_{(3)}\end{array}$ \\
\hline II & 6 & 83157 & 29935 & $\begin{array}{lll}7 & 0 & 4\end{array}$ & 646 & 224 \\
\hline III & 6 & $7417 \quad 3$ & 31228 & $5 \quad 12$ & $\begin{array}{lll}13 & 8 & 6\end{array}$ & $0 \quad 25$ \\
\hline I & 9 & & & 56505 & 19150 & $3132_{(7)}$ \\
\hline
\end{tabular}

Tab. 2.

Anm. Die jeweils erste Zahl entspricht dem Index 4, die zweite Zahl dem Index 4/5, die dritte Zahl dem Index 5 ; die in Klammer gesetzten Zahlen entsprechen den unbestimmbaren Fällen. 


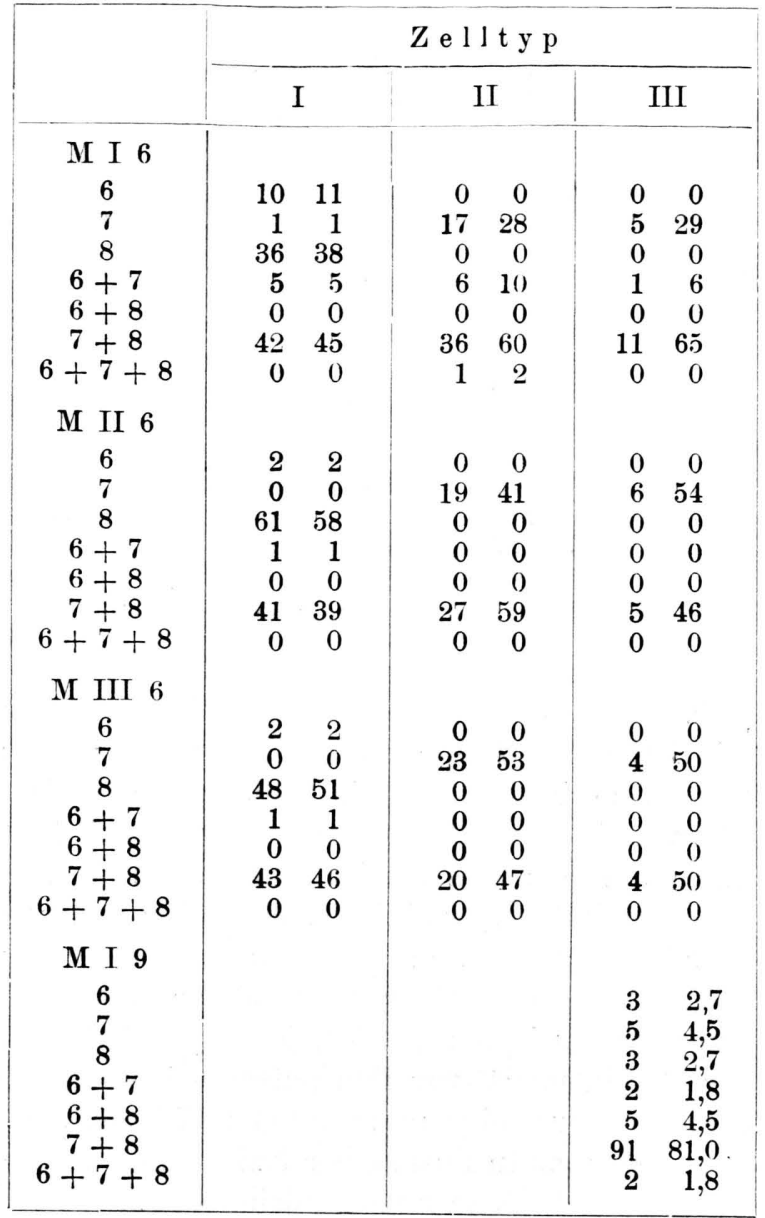

Tab. 3. Vergleich der Formen des Nucleolarchromatins. Die erste Zahl gibt den absoluten Wert, die zweite den Prozentsatz an.

chen aus Nucleolarchromatin liegt an und in der Kernmembran (Anlagerung + Einlagerung), ohne daß noch die Beziehungen zu einer gröberen Nucleolarmasse im Inneren des Kernes gegeben sind.

Zur Beleuchtung der Leistungsfähigkeit unserer Methode und der Erwartungen, die man fernerhin an sie knüpfen kann, seien folgende zusammenfassende Zahlen kurz diskutiert.

1. Vergleich der Hauptzelltypen: Aus der Tab.2a ergibt sich eine relativ gute Übereinstimmung des Verhaltens der Zelltypen in Areal 6 der Maus in drei verschiedenen Gehirnserien (Maus I: Weibchen, $13 \mathrm{~g}$, etwa 7 Wochen alt; Maus II: Männchen, etwa $21 \mathrm{~g}$; Maus III: Weibchen, etwa $17 \mathrm{~g}$ ). Dagegen ergibt die Auszählung des Areales 9 (Nucleus suprachiasmaticus) bei Maus I eine starke Abwandlung des Bildes. Die Kernmes-

\begin{tabular}{|c|c|c|c|c|c|}
\hline & & \multicolumn{4}{|c|}{$\mathrm{Ze} 11 \mathrm{ty} \mathrm{p}$} \\
\hline & & I & II & III & IV \\
\hline $\begin{aligned} 6_{29} & \text { M I } \\
& \text { M II } \\
& \text { M III } \\
& \text { M I }\end{aligned}$ & $\begin{array}{l}6 \\
6 \\
6 \\
9\end{array}$ & $\begin{array}{l}0,09 \\
0,03 \\
0,03\end{array}$ & $\begin{array}{l}0,12 \\
0 \\
0\end{array}$ & $\begin{array}{l}0 \\
0 \\
0 \\
0,04\end{array}$ & $\begin{array}{l}0 \\
0 \\
0,05 \\
0,06\end{array}$ \\
\hline $\begin{array}{ll}7_{29} & \text { M I } \\
\text { M II } \\
\text { M III } \\
\text { M I }\end{array}$ & $\begin{array}{l}6 \\
6 \\
6 \\
9\end{array}$ & $\begin{array}{l}0,44 \\
0,26 \\
0,21\end{array}$ & $\begin{array}{l}0,78 \\
0,33 \\
0,21\end{array}$ & $\begin{array}{l}0,53 \\
0,36 \\
0,25 \\
0,26\end{array}$ & $\begin{array}{l}0,90 \\
0,69 \\
0,27 \\
0,50\end{array}$ \\
\hline $\begin{array}{ll}7_{15} & \text { M I } \\
\text { M II } \\
\text { M III } \\
\text { M I }\end{array}$ & $\begin{array}{l}6 \\
6 \\
6 \\
9\end{array}$ & $\begin{array}{l}0,67 \\
0,25 \\
0,35\end{array}$ & $\begin{array}{l}1,22 \\
3,39 \\
3,05\end{array}$ & $\begin{array}{l}3,0 \\
1,64 \\
3,0 \\
0,97\end{array}$ & $\begin{array}{l}4,9 \\
2,62 \\
2,8 \\
0,88\end{array}$ \\
\hline $\begin{array}{ll}\bar{\tau}_{17} & \text { M I } \\
\text { M II } \\
\text { M III } \\
\text { M I }\end{array}$ & $\begin{array}{l}6 \\
6 \\
6 \\
9\end{array}$ & $\begin{array}{l}0,04 \\
0,04 \\
0,04\end{array}$ & $\begin{array}{l}0,17 \\
0,28 \\
0,26\end{array}$ & $\begin{array}{l}0,53 \\
0,1 \\
0,26 \\
0,65\end{array}$ & $\begin{array}{l}0,1 \\
0 \\
0,32 \\
0,63\end{array}$ \\
\hline $\begin{array}{ll}10_{29} & \text { M I } \\
\text { M II } \\
\text { M III } \\
\text { M I }\end{array}$ & $\begin{array}{l}6 \\
6 \\
5 \\
9\end{array}$ & $\begin{array}{l}0,33 \\
0,63 \\
0,51\end{array}$ & $\begin{array}{l}0,02 \\
0,02 \\
0,02\end{array}$ & $\begin{array}{l}0 \\
0,18 \\
0 \\
0,23\end{array}$ & $\begin{array}{l}0 \\
0,06 \\
0 \\
0,2\end{array}$ \\
\hline $\begin{array}{ll}10_{15} & \text { M I } \\
\text { M II } \\
\text { M III } \\
\text { M I }\end{array}$ & $\begin{array}{l}6 \\
6 \\
6 \\
9\end{array}$ & $\begin{array}{l}0,15 \\
0,20 \\
0,22\end{array}$ & $\begin{array}{l}0,78 \\
0,33 \\
0,07\end{array}$ & $\begin{array}{l}0,12 \\
0,36 \\
0,38 \\
0,52\end{array}$ & $\begin{array}{l}0,40 \\
0,31 \\
0 \\
0,2 \check{5}\end{array}$ \\
\hline $\begin{array}{ll}11_{29} & \text { M I } \\
\text { M II } \\
\text { M III } \\
\text { M I }\end{array}$ & $\begin{array}{l}6 \\
6 \\
6 \\
9\end{array}$ & $\begin{array}{l}0,18 \\
0,06 \\
0,04\end{array}$ & $\begin{array}{l}0,02 \\
0 \\
0\end{array}$ & $\begin{array}{l}0,18 \\
0 \\
0,12 \\
0,05\end{array}$ & $\begin{array}{l}0 \\
0 \\
0 \\
0,06\end{array}$ \\
\hline $\begin{array}{ll}11_{15} & \text { M I } \\
& \text { M II } \\
& \text { M III } \\
& \text { M I }\end{array}$ & $\begin{array}{l}6 \\
6 \\
6 \\
9 \\
9\end{array}$ & $\begin{array}{l}0,20 \\
0,01 \\
0,07\end{array}$ & $\begin{array}{l}0,13 \\
0,15 \\
0,11\end{array}$ & $\begin{array}{l}0,20 \\
0 \\
0 \\
0,08\end{array}$ & $\begin{array}{l}0,1 \\
0 \\
0,0.5 \\
0,06\end{array}$ \\
\hline
\end{tabular}

Tab. 4. Vergleich der Lagerung des Nucleolarchromatins.

sungsergebnisse werden in ausführlichen Arbeiten dargestellt und diskutiert werden.

2. Vergleich der Cytoplasmaverhältnisse: Aus Tab. 2 b ergibt sich, daß im Zelltyp I in allen drei Fällen die Zellen mit dunklem Cytoplasma überwiegen, in Zelltyp II dagegen die mit hellem Plasma. Zelltyp III läßt nicht mehr derart einseitige Entscheidungen zu.

3. Vergleich der Formen des Nucleolarchromatins: Aus Tab. 3 ergibt sich, daß wiederum die Úbereinstimmung in bezug auf ein gleiches Area! überraschend deutlich hervortritt. Es ist dies zugleich für uns ein Beweis, daß die von uns aufgestellten Zelltypen tatsächlich nicht nur eine qualitative, sondern darüber hinaus eine wohl zum erstenmal gefaßte quantitative Differenzierung darstellen. So stehen bei Zelltyp I die Emissionen 
(Symbol 8) ganz im Vordergrund, während z. B. Zelltyp II auch recht oft im rein emittierten $\mathrm{Zu}$ stand erscheint (Symbol 7). Interessant ist das Ergebnis bei Zelltyp III, wo erst durch die gröBere Zahl der zu diesem Typ zu rechnenden Zellen in Areal 9 die starke Betonung der Kombination $7+8$ (s. o.) deutlich wird.

4. Vergleich der Lagerung des Nucleolarchromatins: In Tab. 4 habe ich einige Möglichkeiten dieser Lagerung aus unseren Protokollen herausgezogen. Auf die absoluten Werte ist verzichtet, es ist vielmehr die Häufigkeit eines bestimmten Lagerungstypus pro Zelle errechnet. Der Wert 1 würde also besagen, daß die diskutierte Nucleolarlagerung in jeder der 200 untersuchten Zellen zu erwarten wäre. Auch diese Werte ergeben, zumindest nach der Größenordnung, eine auffallende Übereinstimmung in den Arealen. Im einzelnen können wir aus unseren Werten schließen, daß wir den gewöhnlichen Typ des Nucleolus bei der Maus in keinem Zelltyp und in keinem Areal auch nur einigermaßen sicher antreffen $\left(6_{29}\right)$. Die Mengen des zersplitterten (emittierten) Nucleolarchromatins (7) spielen dagegen sowohl frei im Zellkern gelagert (Index 29) wie auch in Form kleiner An- und Einlagerungen (Index 15) oder auch in Form von kleinen An-, Ein- und Auf- lagerungen (Index 17) eine viel beträchtlichere Rolle. Ganz besonders deutliche Unterschiede ergeben sich dabei zwischen den Zellkernen der einzelnen Zelltypen. Die bereits bei der qualitativen Untersuchung erkannten Differenzen zwischen Zelltyp I und II spiegeln sich in den Zahlenwerten deutlich wieder. Der zentral im Zellkern gelegene Nucleolarapparat mit den Zeichen der Emission $\left(10_{29}\right)$ findet sich in erster Linie bei Zelltyp I, das kernwandständige emittierte $\mathrm{Nu}$ cleolarchromatin dagegen besonders bei Zelltyp II. Es würde indessen zu weit führen, alle in der Tab. 4 enthaltenen Zahlenwerte durchzusprechen.

Soweit dürfen aber die Ergebnisse präzisiert werden: Neben der rein qualitativen Cytoarchitektonik ist durch unsere Methode zum erstenmal eine über die Karyoarchitektonik durchgeführte quantitative Analyse eines Hirnareales erreicht. $\mathrm{Ob}$ die Ergebnisse lediglich im morphologischen Sinn dargestellt werden dürfen, oder ob hinter ihnen auch etwas für die physiologische Analyse des Hypothalamus Bedeutungsvolles steht, können wir noch nicht sicher entscheiden.

Die Arbeit wurde durchgeführt mit Hilfe einer großzügigen Unterstützung durch die LeibnizStiftung für Kunst und Wissenschaft, wofür ich meinen Dank auch hier aussprechen möchte.

\title{
Ergebnisse einer Untersuchung über das Schillern von Federn ${ }^{1}$
}

\author{
Von Wilhelm Josef Schmidt \\ Aus dem Zoologischen Institut der Universität Gießen \\ (Z. Naturforschg. 3 b, 55-57 [1948]; eingegangen am 29. November 1947)
}

$\mathrm{D}$ as Schillern des Gefieders, bei so manchen Vögeln verbreitet, verlockt durch seine Schönheit zu immer erneuter Prüfung, sei es der physikalischen Ursachen, deren morphologischer Grundlagen oder der Bedeutung, die solcher Schmuck im Leben seines Trägers hat. Hinsichtlich des optischen Zustandekommens des Schillerns war der letzte Untersucher, Elsässer², vor allem auf Grund der Beziehungen zwischen Farbe, Polarisationszustand, Phasenunterschied des reflektierten und Winkel des einfallenden Lichtes zu dem Schluß gekommen, ein festes dünnes Blätt-

1 Hrn. Prof. Dr. August Reichensperger in Bonn zum 70. Geburtstage (8. 1. 1948).

2 Th. Els äs s er, Die Struktur schillernder Federn. J. Ornithol. 73, 337-389 [1925]; s. hier auch das ältere Schrifttum. chen erzeuge das Schillern; seine Wirksamkeit sei aber an die Anwesenheit von Melanin gebunden, das einen schwarzen Hintergrund abgebe ${ }^{3}$. Das Blättchen sah Elsässer unter Zuziehung mikroskopischer Befunde in dem Hornmantel schillernder Zellen (der Federradien), der nicht nur die erforderliche (geringe) Dicke habe, sondern auch (bei Lophophorus) die ungemein hohe Brechzahl 1,75-1,78, die sich nach dem Brewsterschen Gesetz ergab.

Eine sorgsame Untersuchung der einschlägigen Verhältnisse im durchfallenden — gewöhnlichen und polarisierten -, weiter im auffallenden Licht

3 Wie Seifenblasen und im Tierreich z. B. die prachtvoll schillernden glasklaren Sapphirinen bezeugen, ist ein dunkler Hintergrund für Farben dünner Blättchen keineswegs erforderlich. 